\title{
On the third natural representation module $M(n-3,3)$ of the permutation groups
}

\section{Ali Abdul Sahib M.Al-Butahi Department of Mathematics and computer applications College of Sciences}

\section{Al-Nahrian University alibotahi@gmail.com}

Recived : $2 \backslash 4 \backslash 2018$

Revised : 9\4\2018

\author{
Reyadh Delfi Ali \\ Department of Mathematics \\ College of Education for \\ Pure Science \\ Kerbala University \\ reyadhdelphi@gmail.com
}

Available online : $17 / 5 / 2018$

DOI: 10.29304/jqcm.2018.10.2.386

\begin{abstract}
:
The main purpose of this work is to propose the third natural representation $M(n-3,3)$ of the symmetric groups over a field $\mathbf{F}$ and prove that $M(n-3,3)$ is split iff $\mathrm{p}$ does not divide $\frac{\mathrm{n}(\mathrm{n}-1)(\mathrm{n}-2)}{6}$.
\end{abstract}

Keywords: symmetric group, group algebra $\mathrm{FS}_{\mathrm{n}}, \mathrm{FS}_{\mathrm{n}}$ - module, Spechet module, exact sequence.

Mathematics Classification :20C30.

\section{Introduction}

In 1935, W .Specht introduced tableau correspondence polynomials, known Specht polynomials, that proved how a given polynomial can be written as a linear combination of other polynomials.(see[Kerber:2004]).This was the results of Specht study on representation theory of symmetric groups, after he faced the problem when the symmetric group acts, in natural way, as a tableaux. However, the result of permutation a standard tableau can be a nonstandard tableau and this nonstandard tableau can be written as a linear combination of Specht polynomials. On the other hand, the representation with partition $\mu=(n-1,1)$ for a positive integer $n$, was first studied by
H.K.Farahat in 1962 [Farahat:1962]. This type of representation is called the natural representation. Seven years later, M.H.Peel introduced in [Peel:1969] and [Peel:1971] the second representation of the symmetric groups and renamed Farahat natural representation by the first natural representation .In Peel's representation, the partition was then $\mu=(n-$ 2,2) for a positive integer n. He also represented the $r^{\text {th }}$-Hook representation where the partition $\mu=\left(n-r, 1^{r}\right)$, for any $r \geq 1$.

For the author's knowledge, no one has studied the $3^{\text {rd }}$-natural representation so far . Therefore, this work represent of the symmetric groups over a field $\mathbf{F}$ and $x_{1}, x_{2}, \ldots, x_{n}$ defined to be linearly independent commuting variables over F. 


\section{Preminaries}

Definition 1: Let $X=\left\{x_{1}, x_{2}, \ldots, x_{n}\right\}$ be a finite set, then the symmetric group on $X$ is the group whose elements "permutations" can be viewed as a bijective function from $\mathbf{F}\left[x_{1}, x_{2}, \ldots, x_{n}\right] \quad$ onto $\quad \mathbf{F}\left[x_{1}, x_{2}, \ldots, x_{n}\right]$.The symmetric group on $\mathrm{X}$ is denoted by $\mathbf{S}_{\mathbf{X}}$ or $\mathbf{S}_{\mathbf{n}}$. Then $\mathbf{F S}_{\mathbf{n}}$ is called the group algebra of the symmetric group $\mathbf{S}_{\mathbf{n}}$ with respect to addition of functions, composition of functions and product of functions by scalars [Joyce:2008].

Definition 2: Let $\mathrm{n}$ be a natural number then the sequence $\mu=\left(\mu_{1}, \mu_{2}, \ldots, \mu_{l}\right)$ is called a partition of $n$ if $\mu_{1} \geq \mu_{2} \geq \cdots \geq \mu_{l}>0$ and $\mu_{1}+\mu_{2}+\cdots+\mu_{l}=n$, the set $D_{\mu}=$ $\left\{(i, j) \mid i=1,2, \ldots, l ; 1 \leq j \leq \mu_{i}\right\}$ is called $\mu-$ diagram and any bijective function $t: D_{\mu} \rightarrow$ $\left\{x_{1}, x_{2}, \ldots, x_{n}\right\}$ is called a $\mu$-tableau. A $\mu-$ tableau may be thought as an array consisting of $l$ rows and $\mu_{1}$ columns of distinct variables $t((i, j))$ where the variables appear in the first $\mu_{i}$ positions of the $i^{\text {th }}$ row and each variable $t((i, j))$ appears in the $i^{\text {th }}$ row and the $j^{\text {th }}$ column $((i, j)$-position $)$ of the array.t $((i, j))$ will be denoted by $t(i, j)$ for each $(i, j) \in D_{\mu}$. The set of all $\mu$-tableaux will be denoted by $T_{\mu}$. i.e $T_{\mu}=\{t \mid t$ is a $\mu-$ tableau $\}$. Then the function $h: T_{\mu} \rightarrow F\left[x_{1}, x_{2}, \ldots, x_{n}\right]$ which is defined by $h(t)=\prod_{i=1}^{l} \prod_{j=1}^{\mu_{i}}(t(i, j))^{i-1}, \forall t \in T_{\mu} \quad$ is called the row position monomial function of $T_{\mu}$, and for each $\mu$-tableau $t, h(t)$ is called the row position monomial of $t$. So $M(\mu)$ is the cyclic $F S_{n}$-module generated by $h(t)$ over $F S_{n}$.[Ellers:2007]

\section{The Third Natural Representation of $S_{n}$}

In the beginning, we determine some denotations which we need them in this paper.

1. Let $\sigma_{1}(n)=\sum_{i=1}^{n} x_{i}$.

2. Let $\sigma_{2}(n)=\sum_{1 \leq i<j \leq n} x_{i} x_{\mathbf{j}}$.

3. Let $\sigma_{3}(n)=\sum_{1 \leq i<j<k \leq n} x_{i} x_{\mathrm{j}} x_{k}$.

4. Let $\quad C_{l}(n)=x_{l}\left(\sigma_{2}(n)-\sum_{\substack{j=1 \\ j \neq l}}^{n} x_{l} x_{\mathrm{j}}\right) ; l=$ $1,2, \ldots, n$. Then $\quad \sum_{i=1}^{n} c_{i_{i}}(n)=\sigma_{3}(n) \quad$ and $\operatorname{dim}_{F}\left(F \sigma_{1}(n)\right)=\operatorname{dim}_{F}\left(F \sigma_{2}(n)\right)=$ $\operatorname{dim}_{F}\left(F \sigma_{3}(n)\right)=1 . \quad F \sigma_{1}(n), F \sigma_{2}(n)$ and $F \sigma_{3}(n)$ are all $F S_{n}$-modules, since $\tau \sigma_{k}(n)=$ $\sigma_{k}(n) \forall k=1,2,3$.

5. Let $u_{i j}(n)=C_{i}(n)-C_{j}(n) ; i, j=$ $1,2, \ldots, n$.
We denote $\bar{V}$ to be the $F S_{n}$-modules generated by $C_{1}(n)$ over $F S_{n}$ and $\bar{V}_{0}$ to be the $F S_{n^{-}}$ submodule of $\bar{V}$ generated by $u_{12}(n)$ over $S_{n}$.

Definition3.1: The $F S_{n}$-module $M(n-r, r)$ defined by

$$
M(n-r, r)=F S_{n} x_{1} x_{2} \ldots x_{r} \quad ;
$$

$n \geq r$

is called $\mathrm{r}^{\text {th }}$ - natural representation module of $S_{n}$ over F.

Lemma3.2: The set $B(n-3,3)=\left\{x_{i} x_{j} x_{l}: 1 \leq\right.$ $i<j<l \leq n\}$ is a F-basis of $M(n-3,3)$ and $\operatorname{dim}_{F} M(n-3,3)=\left(\begin{array}{c}n \\ 3\end{array}\right) ; n \geq 3$.

\section{Proof: Clear}

Theorem3.3: The set

$B_{0}(n-3,3)=\left\{x_{i} x_{j} x_{l}-x_{1} x_{2} x_{3}: 1 \leq i<j<\right.$ $l \leq n,(i, j, l) \neq(1,2,3)\}$ is a F-basis of $M_{0}(n-$ 3,3) and $\operatorname{dim}_{F} M_{0}(n-3,3)=\left(\begin{array}{l}n \\ 3\end{array}\right)-1 ; n \geq 3$.

Proof: Since $M_{0}(n-3,3)=\left\{\sum_{1 \leq i<j<l \leq n} k_{i j l} x_{i} x_{\mathrm{j}} x_{l}\right.$ : $\sum_{i<j<l \leq n} k_{i j l}=0$ and $\left.k_{i j l} \in F\right\}$, we get that $B_{0}(n-$ $3,3) \subset M_{0}(n-3,3)$.To prove $B_{0}(n-3,3)$ generates $M_{0}(n-3,3)$ over $\mathrm{F}$.

Let $x \in M_{0}(n-3,3) . \Rightarrow x=$

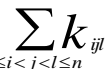

$x_{i} x_{\mathrm{j}} x_{l} ; \sum_{1 \leq i<j<l \leq n} k_{i j l}=0$

$\Rightarrow x=\sum_{1 \leq i<j<l \leq n} k_{i j l} x_{i} x_{\mathrm{j}} x_{l}-0 . x_{1} x_{2} x_{3}$

$\Rightarrow x=\sum_{1 \leq i<j<l \leq n} k_{i j l} x_{i} x_{\mathrm{j}} x_{l}-\left(\sum_{1 \leq i<j<l \leq n} k_{i j l}\right) x_{1} x_{2} x_{3}$

$\Rightarrow x=\sum_{1 \leq i<j<l \leq n} k_{i j l} x_{i} x_{j} x_{l}-\sum_{1 \leq i<j<l \leq n} k_{i j l} x_{1} x_{2} x_{3}$

$\Rightarrow x=\sum_{1 \leq i<j<l \leq n} k_{i j l}\left(x_{i} x_{\mathrm{j}} x_{l}-x_{1} x_{2} x_{3}\right) \quad$ with the

term 123 excluded from the summation since $k_{i j l}\left(x_{1} x_{2} x_{3}-x_{1} x_{2} x_{3}\right)=0$.Hence $B_{0}(n-3,3)$ generates $M_{0}(n-3,3)$ over $\mathrm{F}$.Moreover $B_{0}(n-3,3)$ is linearly independent since if

$$
\begin{array}{cl} 
& \sum_{1 \leq i<j<l \leq n} k_{i j l}\left(x_{i} x_{\mathrm{j}} x_{l}-x_{1} x_{2} x_{3}\right)=0 \quad \Rightarrow \\
& \sum_{1 \leq i<j<l \leq n} k_{i j l} x_{i} x_{\mathrm{j}} x_{l}-\sum_{1 \leq i<j<l \leq n} k_{i j l} x_{1} x_{2} x_{3}=0 \\
\Rightarrow & \sum_{1 \leq i<j<l \leq n} k_{i j l} x_{i} x_{\mathrm{j}} x_{l}=0 \text {,where } \quad k_{123}=- \\
\sum_{1 \leq i<j<l \leq n} k_{i j l} \text { with } \sum_{1 \leq i<j<l \leq n} k_{i j l}=0 \text { and } \quad(i, j, l) \neq
\end{array}
$$
$(1,2,3)$. By lemma (3.2) we have $B(n-3,3)$ is linearly independent. Thus we get $k_{i j l}=$ $0 \forall i, j, l ; 1 \leq i<j<l \leq n$. Hence $B_{0}(n-$ $3,3)$ is a F-basis of $M_{0}(n-3,3)$ and $\operatorname{dim}_{F} M_{0}(n-3,3)=\left(\begin{array}{l}n \\ 3\end{array}\right)-1 ; n \geq 3$ 
Theorem3.4: The set $B=\left\{C_{i}(n) \mid i=1,2, \ldots, n\right\}$ is a F-basis for $\bar{V}(n)=F S_{n} C_{1}(n)$.

Proof: Let $\tau_{i}=\left(x_{1} x_{i}\right) \in S_{n} ; 1<i \leq n$. Then $\tau_{i}\left(C_{1}(n)\right)=C_{i}(n) ; i=1,2, \ldots, n$.

Thus $C_{i}(n) \in \bar{V}(n) ; i=1,2, \ldots, n$.Hence $\mathrm{B}$ $\subset \bar{V}(n)$.

Now

$w \in \bar{V}(n) . \Longrightarrow w=\sum_{i=1}^{(n-1) !} \sum_{j=1}^{n} k_{i j} \tau_{i j} C_{1}(n)$

where $\tau_{i j} \in S_{n}, k_{i j} \in F$ and

$\tau_{i j}\left(x_{1}\right)=x_{j}$, which implies that $\tau_{i j}\left(C_{1}(n)\right)=$ $C_{j}(n) ; j=1,2, \ldots, n$.

$\Rightarrow w=\sum_{i=1}^{(n-1) !} \sum_{j=1}^{n} k_{i j} \tau_{i j} C_{1}(n)$

$=\sum_{j=1}^{n}\left(\sum_{i=1}^{(n-1) !} k_{i j}\right) C_{j}(n)=\sum_{j=1}^{n} d_{j} C_{j}(n)$

,where $\quad d_{j}=\sum_{i=1}^{(n-1) !} k_{i j} \quad$ Hence

generates $\bar{V}(n)=F S_{n} C_{1}(n)$ over $\mathrm{F}$.

If $\quad \sum_{i=1}^{n} k_{i} C_{i}(n)=0 . \Rightarrow k_{1} C_{1}(n)+k_{2} C_{2}(n)+$ $\cdots+k_{n} C_{n}(n)=0$.

$\Rightarrow k_{1}+k_{2}+\cdots+k_{n}=0 \quad$ since $\quad C_{l}(n)=$ $\sum_{1 \leq i<j<l \leq n} x_{i} x_{\mathrm{j}} x_{l}$. Thus $\mathrm{B}$ is a linearly independent. Therefore B is a basis of $\bar{V}(n)$ and $\operatorname{dim}_{F} \bar{V}(n)=n$.

Theorem3.5: $\bar{V}(n)=F S_{n} C_{1}(n)$ and $M(n-$ $1,1)$ are isomorphic over $F S_{n}$.

Proof: Let $\varphi: M(n-1,1) \rightarrow \bar{V}(n)$ be defined as follows:

$\varphi\left(x_{i}\right)=C_{i}(n) ; i=1,2, \ldots, n$. Then for each $\tau=\left(x_{i} x_{j}\right) \in S_{n}$ such that $\tau\left(x_{i}\right)=x_{j}$ we get that $\varphi\left(\tau x_{i}\right)=\varphi\left(x_{j}\right)=C_{j}(n) \quad=\tau C_{i}(n)=$ $\tau \varphi\left(x_{i}\right)$.Hence $\varphi$ is a $F S_{n^{-}}$ homomorphism .Also $y=$ $\sum_{i=1}^{n} k_{i} C_{i}(n)$ for any $y \in \bar{V}$.Thus for all $y \in \bar{V}, \exists w=\sum_{i=1}^{n} k_{i} x_{i} \in M(n-$

1,1) such that $\quad \varphi(w)=\varphi\left(\sum_{i=1}^{n} k_{i} x_{i}\right)$

$=\sum_{i=1}^{n} \varphi\left(k_{i} x_{i}\right)=\sum_{i=1}^{n} k_{i} \varphi\left(x_{i}\right)=$

$\sum_{i=1}^{n} k_{i} C_{i}(n)=y$. Hence $\varphi$ is an epimorphism.

Thus $\quad \operatorname{dim}_{F} \operatorname{ker} \varphi=\operatorname{dim}_{F} M(n-1,1)-$ $\operatorname{dim}_{F} \bar{V}=n-n=0 . \operatorname{ker} \varphi=0$. Then $\varphi$ is a monomorphism .

Thus $\varphi$ is a $F S_{n}-$ isomorphism. Hence $M(n-1,1)$ and $\bar{V}$ are isomorphic over $F S_{n}$.

Theorem3.6: If $p$ does not divides $\mathrm{n}$, then $\bar{V}(n)=\bar{V}_{0}(n) \oplus F \sigma_{3}(n)$
Proof : From Theorem (3.5) we have a $F S_{n}-$ isomorphism

$\varphi: M(n-1,1) \rightarrow \bar{V}(n)$ such that $\varphi\left(x_{i}\right)=$ $C_{i}(n) ; i=1,2, \ldots, n$.

And since $M_{0}(n-1,1)=F S_{n}\left(x_{2}-x_{1}\right) \subset$ $M(n-1,1)$, then $\psi=\left.\varphi\right|_{M_{0}(n-1,1)}$ is a $F S_{n}-$ isomorphism. Thus $\bar{V}_{0}(n)$ and $M_{0}(n-1,1)$ are isomorphic over $F S_{n}$ which is irreducible submodule over $F S_{n}$ when $p$ does not divides $n$ and $\sigma_{3}(n) \notin \bar{V}_{0}(n)$ when $p$ does not divide $n$ since the sum of the coefficients of the $C_{i}(n)$ in $\sigma_{3}(n)$ is $n$. Hence $\bar{V}_{0}(n) \cap F \sigma_{3}(n)=0$, $F \sigma_{3}(n) \subset \bar{V}(n)$ and $\bar{V}_{0}(n) \subset \bar{V}(n)$. But $\operatorname{dim}_{F} \bar{V}_{0}(n)+\operatorname{dim}_{F} F \sigma_{3}(n)=n-1+1=n=$ $\operatorname{dim}_{F} \bar{V}(n)$

Hence $\bar{V}_{0}(n) \oplus F \sigma_{3}(n)=\bar{V}(n)$ when $p$ does not divides $n$.

Proposition 3.7 : If $p$ does not divides $n$, then $\bar{V}$ has the following two composition series $0 \subset \bar{V}_{0}(n) \subset \bar{V}(n)$ and $0 \subset F \sigma_{3}(n) \subset \bar{V}(n)$.

Proof : Since $p$ does not divides $n$, then by Theorem (3.6) we have

$\bar{V}=\bar{V}_{0}(n) \oplus F \sigma_{3}$, and $\bar{V}_{0}(n)$ is irreducible submodule when $p$ does not divide $n$. Hence $\frac{\bar{V}}{F \sigma_{3}(n)}=\frac{\bar{V}_{0}(n) \oplus F \sigma_{3}(n)}{F \sigma_{3}(n)} \simeq \bar{V}_{0}(n)$. Thus $\frac{\bar{V}}{F \sigma_{3}(n)}$ is irreducible module when $p$ does not divide $n$. Since $\operatorname{dim}_{F} F \sigma_{3}(n)=1$. Then $F \sigma_{3}(n)$ is irreducible submodule over $F S_{n}$. But $\frac{\bar{V}}{\bar{V}_{0}(n)}=$ $\frac{\bar{V}_{0}(n) \oplus F \sigma_{3}(n)}{\bar{V}_{0}(n)} \simeq F \sigma_{3}(n)$. Therefore $\frac{\bar{V}}{\bar{V}_{0}(n)}$ is irreducible module over $F S_{n}$. Thus we get the following two composition series $\bar{V}$ $0 \subset \bar{V}_{0}(n) \subset \bar{V}$ and $0 \subset F \sigma_{3}(n) \subset$

Theorem 3.8: The following sequence

$$
\begin{aligned}
O \rightarrow M_{0}(n-3,3) & \stackrel{i}{\rightarrow} M(n-3,3) \stackrel{f}{\rightarrow} F \\
& \rightarrow O
\end{aligned}
$$

over a field $F$ is split iff $p$ does not divide $\frac{n(n-1)(n-2)}{6}$.

Proof: If $p$ does not divide $\frac{n(n-1)(n-2)}{6}$. For any $k \in F \quad$ we have $f\left(\sum_{1 \leq i<j<l \leq n} k_{i j l} x_{i} x_{\mathrm{j}} x_{l}\right)=$ $\sum_{1 \leq i<j<l \leq n} k_{i j l}=k$.Hence $f$ is on to. Moreover 
Ali .A/Reyadh .D

$$
\begin{aligned}
& \text { kerf }=\left\{\sum_{1 \leq i<j<l \leq n} k_{i j l} x_{i} x_{\mathrm{j}} x_{l}:\right. \\
& \left.f\left(\sum_{1 \leq i<j<l \leq n} k_{i j l} x_{i} x_{\mathrm{j}} x_{l}\right)=0\right\} \\
& \left\{\sum_{1 \leq i<j<l \leq n} k_{i j l} x_{i} x_{\mathrm{j}} x_{l}: \sum_{1 \leq i<j<l \leq n} k_{i j l}=0 \quad\right\}=
\end{aligned}
$$$$
M_{0}(n-3,3)=\operatorname{Im} i \text {.Hence the sequence (1) is }
$$
an exact sequence.

So we can defined a function $h: F \rightarrow M(n-$ $3,3)$ by $h(k)=\frac{6 k \sigma_{3}(n)}{n(n-1)(n-2)}$ which is a $F S_{n}-$ homomorphism since

$\sum_{\tau \in S_{n}} r \tau h(k)=\quad \sum_{\tau \in S_{n}} r \tau\left(\frac{6 k \sigma_{3}(n)}{n(n-1)(n-2)}\right)=$ $\sum_{\tau \in S_{n}} \frac{6 r k \tau \sigma_{3}(n)}{n(n-1)(n-2)} \quad=\quad \sum_{\tau \in S_{n}} \frac{6 r k \sigma_{3}(n)}{n(n-1)(n-2)}$ $=\sum_{\tau \in S_{n}} r h(k)=h\left(\sum_{\tau \in S_{n}} r k\right)=$ $h\left(\sum_{\tau \in S_{n}} r \tau(k)\right)$.And since $f h(k)=f\left(\frac{6 k \sigma_{3}(n)}{n(n-1)(n-2)}\right)=$ $\frac{6 k}{n(n-1)(n-2)} f\left(\sigma_{3}(n)\right)=$

$$
\frac{6 k}{n(n-1)(n-2)} f\left(\sum_{1 \leq i<j<l \leq n} k_{i j l} x_{i} x_{\mathrm{j}} x_{l}\right)=
$$

$\frac{6 k}{n(n-1)(n-2)} \cdot \frac{n(n-1)(n-2)}{6}=k$.Hence $f h=I$ on

F. Thus the sequence(1) is split.

Now assume the sequence (1) is split.

Then there exist a $F S_{n}$-homomorphism $f_{1}: F \rightarrow M(n-3,3)$ s.t. $f f_{1}=I$ on $\mathrm{F}$.

Let $f_{1}(1)=\sum_{1 \leq i<j<l \leq n} k_{i j l} x_{i} x_{\mathrm{j}} x_{l}$. Then $\tau f_{1}(1)=$ $f_{1}(\tau(1))=f_{1}(1)$, where $\tau=\left(x_{r} x_{s}\right) \in S_{n}, 1 \leq$ $r<s \leq n$. Thus $f_{1}(1)-\tau f_{1}(1)=0$.

$\Rightarrow 0=\sum_{1 \leq i<j<l \leq n} k_{i j l} x_{i} x_{\mathrm{j}} x_{l}-\sum_{1 \leq i<j<l \leq n} k_{i j l} \tau\left(x_{i} x_{\mathrm{j}} x_{l}\right)$

$=\sum_{1 \leq i<j<l \leq n} k_{i j l}\left(x_{i} x_{\mathrm{j}} x_{l}-\tau\left(x_{i} x_{\mathrm{j}} x_{l}\right)\right)$

$$
\begin{gathered}
\sum_{\substack{r+1<j<l \leq n \\
j, l \neq s}} k_{i j l}\left(x_{r} x_{\mathrm{j}} x_{l}-x_{s} x_{\mathrm{j}} x_{l}\right)+ \\
\sum_{\substack{i=1 \\
r-1}}^{n} \sum_{\substack{l=r+1 \\
l \neq s}}^{n} k_{i r l}\left(x_{i} x_{\mathrm{r}} x_{l}-x_{i} x_{\mathrm{s}} x_{l}\right)+
\end{gathered}
$$$$
\sum_{1 \leq i<j<r} k_{\ddot{j} r}\left(x_{i} x_{\mathrm{j}} x_{r}-x_{i} x_{\mathrm{j}} x_{s}\right)=
$$$$
\sum_{\substack{j=r+1 \\ j<l \leq n}}^{n-1}\left(k_{r j l}-k_{s j l}\right) x_{r} x_{\mathrm{j}} x_{l}+
$$$$
\sum_{i=1}^{r-1} \sum_{\substack{l=r+1 \\ l \neq s}}^{n}\left(k_{i r l}-k_{i s l}\right) x_{i} x_{\mathrm{r}} x_{l}+
$$$$
\sum_{1 \leq i<j<r}\left(k_{i j r}-k_{i s l}\right) x_{i} x_{\mathrm{j}} x_{r} .
$$

So by equaling the coefficient, we get $k_{r j l}=k_{s j l} \forall r<j<l \leq n$,

$k_{\text {irl }}=k_{i s l} \quad \forall 1 \leq i<r$ and $r<l \leq n, l \neq s$

$k_{i j r}=k_{i j s} \forall 1 \leq i<j<r$.

Hence for each $r, s$ such that $1 \leq r<s \leq$ $n$ we get

$k_{i j l}=k ; 1 \leq i<j<l \leq n \quad$.Then $f_{1}(1)=$

$\sum_{\leq i<j<l \leq n} k_{i j l} x_{i} x_{\mathrm{j}} x_{l}=\sum_{1 \leq i<j<l \leq n} k x_{i} x_{\mathrm{j}} x_{l}=k \sigma_{3}(n)$.

$\because f f_{1}=I$

$\therefore 1=f f_{1}(1)=f\left(k \sigma_{3}(n)\right)=k f\left(\sigma_{3}(n)\right)=$ $k f\left(\sum_{1 \leq i<j<k \leq n} x_{i} x_{\mathrm{j}} x_{k}\right)=k \frac{n(n-1)(n-2)}{6}$.Hence $\mathrm{p}$ does not divide $\frac{n(n-1)(n-2)}{6}$.

Corollary3.9: $\mathrm{M}(\mathrm{n}-3,3)$ is a direct sums of $M_{0}(n-3,3)$ and $F \sigma_{3}(n)$ when $\mathrm{p}$ does not divide $\frac{n(n-1)(n-2)}{6}$.

Proof: Since $\mathrm{p}$ does not divide $\frac{n(n-1)(n-2)}{6}$,then the sequence (1) is split .Thus $M(n-3,3)$ decomposable into kerf $=M_{0}(n-$ 3,3) and $\operatorname{Imf}=F \sigma_{3}(n)$ since for each $k \sigma_{3}(n) \in F \sigma_{3}(n)$ we have $f\left(\frac{k n(n-1)(n-2)}{6}\right)=$ $\frac{k n(n-1)(n-2)}{6}\left(\frac{6 \sigma_{3}(n)}{n(n-1)(n-2)}\right)=k \sigma_{3}(n)$.

Hence $\mathrm{M}(\mathrm{n}-3,3)=M_{0}(n-3,3) \oplus F \sigma_{3}(n)$.

Theorem 3.10: The following sequence

$$
0 \rightarrow \operatorname{ker} \bar{d}_{2} \underset{0}{\stackrel{i}{\rightarrow}} M_{0}(n-3,3) \stackrel{\bar{d}_{2}}{\rightarrow} M_{0}(n-2,2) \rightarrow
$$

is split iff $\mathrm{p}$ does not divide neither (n-2) nor (n$3)$.

Proof: Since $\bar{d}_{2}\left(\frac{1}{2}\left(x_{1} x_{i} x_{k}-x_{1} x_{2} x_{i}+x_{2} x_{i} x_{k}-\right.\right.$ $\left.\left.x_{1} x_{2} x_{k}\right)\right)=\frac{1}{2}\left(x_{1} x_{i}+x_{1} x_{k}+x_{i} x_{k}-x_{1} x_{2}-\right.$ $x_{1} x_{i}-x_{2} x_{i}+x_{2} x_{i}+x_{2} x_{k}+x_{i} x_{k}-x_{1} x_{2}-$ $\left.x_{1} x_{k}-x_{2} x_{k}\right)=\frac{1}{2}\left(2\left(x_{i} x_{k}-x_{1} x_{2}\right)\right)=x_{i} x_{k}$ $x_{1} x_{2}$.Which is the generated of $M_{0}(n-$ $3,3)$.Hence $\bar{d}_{2}$ is on to map. Moreover $\operatorname{Imi}=$ $\operatorname{ker} \bar{d}_{2}$.Thus the sequence (2) is exact.

If $\mathrm{p}$ does not divide neither (n-2)nor(n-3).

Let $\phi: M_{0}(n-2,2) \rightarrow M_{0}(n-3,3)$ be defined as follows:

$$
\begin{aligned}
& \phi\left(x_{i} x_{j}-x_{1} x_{2}\right)=\frac{1}{(n-2)(n-3)} \sum_{2\langle i<j \leq n}\left(x_{1} x_{i} x_{j}-\right. \\
& \left.x_{1} x_{2} x_{i}+x_{2} x_{i} x_{j}-x_{1} x_{2} x_{j}\right)
\end{aligned}
$$

Then for any $\in S_{n}$, s.t. $\tau\left(x_{1}\right)=x_{1}, \tau\left(x_{2}\right)=$ $x_{2}$.

$$
\begin{aligned}
& \phi\left(\tau\left(x_{i} x_{j}-x_{1} x_{2}\right)\right)=\phi\left(\tau\left(x_{i}\right) \tau\left(x_{j}\right)-\right. \\
& \left.\tau\left(x_{1}\right) \tau\left(x_{2}\right)\right)=\frac{1}{(n-2)(n-3)} \sum_{2\langle i<j \leq n}\left(x_{1} x_{i_{1}} x_{j_{1}}-\right. \\
& \left.x_{1} x_{2} x_{i_{1}}+x_{2} x_{i_{1}} x_{j_{1}}-x_{1} x_{2} x_{j_{1}}\right) \text { Where } \tau\left(x_{i}\right)= \\
& x_{i_{1}} \text { and } \tau\left(x_{j}\right)=x_{j_{1}} \text {.Then }
\end{aligned}
$$


$\phi\left(\tau\left(x_{i} x_{j}-x_{1} x_{2}\right)\right)=$ $\frac{1}{(n-2)(n-3)} \sum_{2\langle i<j \leq n} \tau\left(x_{1} x_{i} x_{j}-x_{1} x_{2} x_{i}+x_{2} x_{i} x_{j}-\right.$ $\left.x_{1} x_{2} x_{j}\right)=\frac{1}{(n-2)(n-3)} \tau \sum_{2\langle i<j \leq n}\left(x_{1} x_{i} x_{j}-x_{1} x_{2} x_{i}+\right.$ $\left.x_{2} x_{i} x_{j}-x_{1} x_{2} x_{j}\right)=\tau \phi\left(x_{i} x_{j}-x_{1} x_{2}\right)$.Hence $\phi$ is a $F S_{n}$-homomorphism .Moreover $\bar{d}_{2} \phi\left(x_{i} x_{j}-x_{1} x_{2}\right)=$ $\bar{d}_{2}\left(\frac{1}{(n-2)(n-3)} \sum_{2\langle i<j \leq n}\left(x_{1} x_{i} x_{j}-x_{1} x_{2} x_{i}+\right.\right.$ $\left.\left.x_{2} x_{i} x_{j}-x_{1} x_{2} x_{j}\right)\right)=$ $\frac{1}{(n-2)(n-3)} \sum_{2\langle i<j \leq n} \bar{d}_{2}\left(x_{1} x_{i} x_{j}-x_{1} x_{2} x_{i}+\right.$ $\left.x_{2} x_{i} x_{j}-x_{1} x_{2} x_{j}\right)=\frac{1}{(n-2)(n-3)} \sum_{2\langle i<j \leq n} 2\left(x_{i} x_{j}-\right.$ $\left.x_{1} x_{2}\right)=\frac{1}{(n-2)(n-3)} \frac{(n-2)(n-3)}{2}\left(2\left(x_{i} x_{j}-\right.\right.$ $\left.\left.x_{1} x_{2}\right)\right)=x_{i} x_{j}-x_{1} x_{2}$

So $\bar{d}_{2} \phi=I$ on $M_{0}(n-2,2)$. Hence the sequence (2) is split if $\mathrm{p}$ does not divide neither (n-2) nor (n-3).Thus $\quad M_{0}(n-3,3)=$ ker $\bar{d}_{2} \oplus L_{0}$, where $L_{0}=\phi\left(M_{0}(n-2,2)\right)$.

Now assume if the sequence (2) is split.

Let $\phi: M_{0}(n-2,2) \rightarrow M_{0}(n-3,3)$ be a $F S_{n}$-homomorphism such that $\bar{d}_{2} \phi=I$. Thus we can define $\phi$ as follows $\phi\left(x_{i_{1}} x_{j_{1}}-x_{1} x_{2}\right)=$ $\sum_{2\langle i<j \leq n} k_{i j}\left(x_{1} x_{i} x_{j}-x_{1} x_{2} x_{i}+x_{2} x_{i} x_{j}-\right.$ $\left.x_{1} x_{2} x_{j}\right) . \Rightarrow \bar{d}_{2} \phi\left(x_{i_{1}} x_{j_{1}}-x_{1} x_{2}\right)=$ $\bar{d}_{2}\left(\sum_{2\langle i<j \leq n} k_{i j}\left(x_{1} x_{i} x_{j}-x_{1} x_{2} x_{i}+x_{2} x_{i} x_{j}-\right.\right.$ $\left.x_{1} x_{2} x_{j}\right)=\sum_{2\langle i<j \leq n} \bar{d}_{2}\left(k_{i j}\left(x_{1} x_{i} x_{j}-x_{1} x_{2} x_{i}+\right.\right.$ $\left.x_{2} x_{i} x_{j}-x_{1} x_{2} x_{j}\right)=\sum_{2\langle i<j \leq n} k_{i j}\left(2\left(x_{i} x_{j}-x_{1} x_{2}\right)\right.$ $=2\left(\sum_{2\langle i<j \leq n} k_{i j}\left(x_{i} x_{j}-x_{1} x_{2}\right)\right)=x_{i_{1}} x_{j_{1}}-x_{1} x_{2}$. $\Rightarrow 2 \sum_{2\langle i<j \leq n} k_{i j}=$ $\begin{cases}0 & \text { if }(i, j) \neq\left(i_{1}, j_{1}\right) \\ 1 & \text { if }(i, j)=\left(i_{1}, j_{1}\right)\end{cases}$

Moreover if $\tau=\left(x_{r} x_{s}\right) \in S_{n} ; 2<r<s \leq n$ such that $\tau\left(x_{i_{1}} x_{j_{1}}\right)=x_{i_{1}} x_{j_{1}}$. Then $\phi\left(\tau\left(x_{i_{1}} x_{j_{1}}-\right.\right.$ $\left.\left.x_{1} x_{2}\right)\right)=\phi\left(x_{i_{1}} x_{j_{1}}-x_{1} x_{2}\right)=\tau \phi\left(x_{i_{1}} x_{j_{1}}-\right.$ $\left.x_{1} x_{2}\right) \Rightarrow \phi\left(x_{i_{1}} x_{j_{1}}-x_{1} x_{2}\right)-\tau \phi\left(x_{i_{1}} x_{j_{1}}-\right.$ $\left.x_{1} x_{2}\right)=0 \Longrightarrow \sum_{2\langle i<j \leq n}\left(k_{i j}\left(x_{1} x_{i} x_{j}-x_{1} x_{2} x_{i}+\right.\right.$ $\left.\left.x_{2} x_{i} x_{j}-x_{1} x_{2} x_{j}\right)\right)-\tau\left(\sum_{2\langle i<j \leq n}\left(k_{i j}\left(x_{1} x_{i} x_{j}-\right.\right.\right.$ $\left.\left.x_{1} x_{2} x_{i}+x_{2} x_{i} x_{j}-x_{1} x_{2} x_{j}\right)\right)=0$.

$$
\begin{aligned}
& \Rightarrow \sum_{\substack{j=r+1 \\
j \neq s}}^{n}\left(k_{r j}-k_{s j}\right) \quad x_{1} x_{r} x_{j} \\
& \sum_{\substack{j=r+1 \\
j \neq s}}^{n}\left(k_{r j}-k_{s j}\right) x_{1} x_{2} x_{r} \\
& \sum_{\substack{j=r+1 \\
j \neq s}}^{n}\left(k_{r j}-k_{s j}\right) x_{2} x_{r} x_{j} \\
& +\sum_{2<i<r}\left(k_{i r}-k_{i s}\right) x_{1} x_{i} x_{r}+\sum_{2<i<r}\left(k_{i r}-k_{i s}\right) x_{1} x_{i} x_{r}+ \\
& \sum_{2<i<r}\left(k_{i r}-k_{i s}\right) x_{2} x_{i} x_{r}-\sum_{2<i<r}\left(k_{i r}-k_{i s}\right) x_{1} x_{2} x_{r}=0
\end{aligned}
$$

$\Rightarrow$ By equaling the above equitation we get $k_{r j}=k_{s j} ; r<j \leq n \& j \neq s$, and $k_{i r}=$ $k_{i s} ; 2<i<r$.

$\Longrightarrow \quad k_{r j}=k_{s j}=k_{i r}=k_{i s}=k$.Thus since $2 \sum_{2\langle i<j \leq n} k_{i j}=0 \quad \Rightarrow \quad 2 \sum_{2\langle i<j \leq n} k=0$ $\Rightarrow 2\left(\begin{array}{c}n-2 \\ 2\end{array}\right) k=(n-2)(n-3) k=0$.Then $k=0$ or $p \mid(n-2)$ or $p \mid(n-3)$.

$\because \quad 2 \sum_{2\langle i<j \leq n} k_{i j}=1$ when $(i, j)=\left(i_{1}, j_{1}\right) \Rightarrow$ $2 \frac{(n-2)(n-3)}{2} k_{1}=1$.

$\Rightarrow p \nmid(n-2), p \nmid(n-3)$ and $k_{1} \neq 0$. Hence we get $p \nmid(n-2), p \nmid(n-3), k=$ 0 and $k_{1} \neq 0$.Thus if the sequence is split then $p$ does not divide neither (n-2) nor (n-3).

Proposition3.11: $S(n-3,3)$ is a proper submodule of $\operatorname{ker} \bar{d}_{2}$ over $F S_{n}$.

Proof: Since $S(n-3,3)=F S_{n}\left(x_{2}-x_{1}\right)\left(x_{4}-\right.$ $\left.x_{3}\right)\left(x_{6}-x_{5}\right)$. i.e.

$\left(x_{2}-x_{1}\right)\left(x_{4}-x_{3}\right)\left(x_{6}-x_{5}\right)$ is the generator of $S(n-3,3)$ over $F S_{n}$, and $\bar{d}_{2}\left(\left(x_{2}-x_{1}\right)\left(x_{4}-\right.\right.$ $\left.\left.x_{3}\right)\left(x_{6}-x_{5}\right)\right)=0$. Hence $S(n-3,3) \subseteq \operatorname{ker} \bar{d}_{2}$. Since $\bar{d}_{2}$ is an epimorphism. Hence

$$
\begin{aligned}
\operatorname{dim}_{F} \operatorname{ker} \bar{d}_{2}=\operatorname{dim}_{F} M_{0}(n-3,3) \\
-\operatorname{dim}_{F} M_{0}(n-2,2) \\
=\frac{n(n-1)(n-2)}{6} \\
-\frac{n(n-1)}{2} \\
=\frac{n(n-1)(n-5)}{6}
\end{aligned}
$$

While $\operatorname{dim}_{F} S(n-3,3)=\frac{n(n-1)}{6}$. Thus $\operatorname{dim}_{F} S(n-3,3)<\operatorname{dim}_{F} \operatorname{ker} \bar{d}_{2}$.

Hence $S(n-3,3)$ is a proper submodule of $\operatorname{ker} \bar{d}_{2}$ over $F S_{n}$. 
Ali .A/Reyadh .D

Proposition3.12: If $p \neq 2,3$ and $\mathrm{p}$ divides $(\mathrm{n}+1)$, then we get the following series: 1) $0 \subset \bar{V}_{0} \subset \bar{V}_{0} \oplus S(n-3,3) \subset \bar{V} \oplus S(n-$ $3,3) \subset \bar{V} \oplus \operatorname{ker} \bar{d}_{2} \subset M_{0}(n-3,3) \oplus F \sigma_{3}$. 2) $0 \subset \bar{V}_{0} \subset \bar{V}_{0} \oplus S(n-3,3) \subset \bar{V}_{0} \oplus \operatorname{ker} \bar{d}_{2} \subset$ $\bar{V} \oplus$ ker $\bar{d}_{2} \subset M_{0}(n-3,3) \oplus F \sigma_{3}$. 3) $\quad 0 \subset \bar{V}_{0} \subset \bar{V} \subset \bar{V} \oplus S(n-3,3) \subset$ $\bar{V} \oplus \operatorname{ker} \bar{d}_{2} \subset M_{0}(n-3,3) \oplus F \sigma_{3}$. 4) $0 \subset F \sigma_{3} \subset \bar{V} \subset \bar{V} \oplus S(n-3,3) \subset$ $\bar{V} \oplus \operatorname{ker} \bar{d}_{2} \subset M_{0}(n-3,3) \oplus F \sigma_{3}$. 5) $0 \subset F \sigma_{3} \subset F \sigma_{3} \oplus S(n-3,3) \subset$ $\bar{V} \oplus S(n-3,3) \subset \bar{V} \oplus \operatorname{ker} \bar{d}_{2} \subset$ $M_{0}(n-3,3) \oplus F \sigma_{3}$. 6) $0 \subset S(n-3,3) \subset F \sigma_{3} \oplus S(n-3,3) \subset$ $\bar{V} \oplus S(n-3,3) \subset \bar{V} \oplus \operatorname{ker} \bar{d}_{2} \subset$ $M_{0}(n-3,3) \oplus F \sigma_{3}$. 7) $0 \subset S(n-3,3) \subset \bar{V}_{0} \oplus S(n-3,3) \subset$ $\bar{V}_{0} \oplus \operatorname{ker} \bar{d}_{2} \subset \bar{V} \oplus \operatorname{ker} \bar{d}_{2} \subset M_{0}(n-3,3) \oplus$ $\mathrm{Fo}_{3}$.

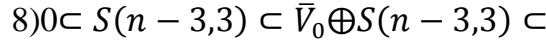
$\bar{V} \oplus S(n-3,3) \subset \bar{V} \oplus \operatorname{ker} \bar{d}_{2} \subset$ $M_{0}(n-3,3) \oplus F \sigma_{3}$.

\section{Proof:}

If $p \neq 2,3$ and $p \mid(n+1)$.

Then $p$ does not divide $n$ nor $(n-$ 1) nor $(n-2)$. Since $\sigma_{3}(n)=$ $\sum_{1 \leq i<j<k \leq n}$ $x_{i} x_{\mathrm{j}} x_{k}$ and the sum of the coefficients is $\frac{n(n-1)(n-2)}{6}, \quad$ then $\quad \sigma_{3}(n) \notin M_{0}(n-$ 3,3).i.e $F \sigma_{3} \cap M_{0}(n-3,3)=0$.which implies that $M(n-3,3)=M_{0}(n-3,3) \oplus$ $F \sigma_{3}$. Moreover we have $S(n-3,3) \subset$ $\operatorname{ker} \bar{d}_{2}$ and $F \sigma_{3}(n) \not \subset \operatorname{ker} \bar{d}_{2}$,thus $F \sigma_{3} \cap S(n-3,3)=0$.

Since $p \nmid n$, then by Theorem (3.6) we have $\bar{V}_{0}(n)$ is an irreducible submodule overFS $S_{n}$, and $\bar{V}=\bar{V}_{0}(n) \oplus F \sigma_{3}$, then $\bar{V} \cap \operatorname{ker} \bar{d}_{2}=0$. Thus $\bar{V} \cap S(n-3,3)=$ 0which implies that $F \sigma_{3} \oplus S(n-$ $3,3) \subset \bar{V} \oplus S(n-3,3)$ and $\bar{V}_{0}(n) \oplus S(n-$ $3,3) \subset \bar{V} \oplus S(n-3,3)$.Therefore we get the following series:

1) $0 \subset \bar{V}_{0} \subset \bar{V}_{0} \oplus S(n-3,3) \subset \bar{V} \oplus S(n-$ $3,3) \subset \bar{V} \oplus \operatorname{ker} \bar{d}_{2} \subset M_{0}(n-3,3) \oplus F \sigma_{3}$. 2) $0 \subset \bar{V}_{0} \subset \bar{V}_{0} \oplus S(n-3,3) \subset \bar{V}_{0} \oplus \operatorname{ker} \bar{d}_{2} \subset$ $\bar{V} \oplus \operatorname{ker} \bar{d}_{2} \subset M_{0}(n-3,3) \oplus F \sigma_{3}$. 3) $\quad 0 \subset \bar{V}_{0} \subset \bar{V} \subset \bar{V} \oplus S(n-3,3) \subset$ $\bar{V} \oplus \operatorname{ker} \bar{d}_{2} \subset M_{0}(n-3,3) \oplus F \sigma_{3}$. 4) $0 \subset F \sigma_{3} \subset \bar{V} \subset \bar{V} \oplus S(n-3,3) \subset$ $\bar{V} \oplus \operatorname{ker} \bar{d}_{2} \subset M_{0}(n-3,3) \oplus F \sigma_{3}$. 5) $0 \subset F \sigma_{3} \subset F \sigma_{3} \oplus S(n-3,3) \subset$ $\bar{V} \oplus S(n-3,3) \subset \bar{V} \oplus \operatorname{ker} \bar{d}_{2} \subset$ $M_{0}(n-3,3) \oplus F \sigma_{3}$.
6) $0 \subset S(n-3,3) \subset F \sigma_{3} \oplus S(n-3,3) \subset$ $\bar{V} \oplus S(n-3,3) \subset \bar{V} \oplus \operatorname{ker} \bar{d}_{2} \subset$ $M_{0}(n-3,3) \oplus F \sigma_{3}$. 7) $0 \subset S(n-3,3) \subset \bar{V}_{0} \oplus S(n-3,3) \subset$ $\bar{V}_{0} \oplus \operatorname{ker} \bar{d}_{2} \subset \bar{V} \oplus \operatorname{ker} \bar{d}_{2} \subset M_{0}(n-3,3) \oplus$ $\mathrm{F}_{3}$.

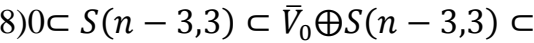
$\bar{V} \oplus S(n-3,3) \subset \bar{V} \oplus$ ker $\bar{d}_{2} \subset$ $M_{0}(n-3,3) \oplus F \sigma_{3}$.

Theorem 3.13: The following sequence of a $F S_{n}$ - modules is short exact sequence.

$0 \rightarrow \operatorname{ker} \overline{\bar{d}}_{2}$

$\stackrel{i}{\rightarrow} G \stackrel{\overline{\bar{d}}_{2}}{\rightarrow} S(n-2,2) \rightarrow 0$

3)

where $G=F S_{n}\left(x_{1} x_{3} x_{6}-x_{1} x_{4} x_{6}+\right.$

$\left.x_{2} x_{4} x_{6}-x_{2} x_{3} x_{6}\right)$

Proof: From the definition of $G$ we get that $G \subset M_{0}(n-3,3)$ and by Theorem(3.10) we have $\bar{d}_{2}: M_{0}(n-3,3) \rightarrow M_{0}(n-2,2)$ is on to map. Since $S(n-2,2)=F S_{n}\left(x_{2}-\right.$ $\left.x_{1}\right)\left(x_{4}-x_{3}\right) \subset M_{0}(n-2,2)$.

Then $\left(x_{2}-x_{1}\right)\left(x_{4}-x_{3}\right)=x_{2} x_{4}-x_{2} x_{3}-$ $x_{1} x_{4}+x_{1} x_{3}$ and $\bar{d}_{2}\left(\left(x_{1} x_{3} x_{6}-x_{1} x_{4} x_{6}+x_{2} x_{4} x_{6}-\right.\right.$ $\left.x_{2} x_{3} x_{6}\right)+\left(x_{1} x_{3} x_{5}-x_{1} x_{4} x_{5}+x_{2} x_{4} x_{5}-\right.$ $\left.\left.x_{2} x_{3} x_{5}\right)\right)=2\left(x_{2} x_{4}-x_{2} x_{3}-x_{1} x_{4}+\right.$ $\left.x_{1} x_{3}\right)=2\left(x_{2}-x_{1}\right)\left(x_{4}-x_{3}\right)$.

Thus $\overline{\bar{d}}_{2}=\left.\bar{d}_{2}\right|_{G}: G \rightarrow S(n-2,2)$ is on to map. Moreover the inclusion map $i$ is oneto-one map and $\operatorname{ker} \overline{\bar{d}}_{2}=I m i$. Hence the sequence (3) is short exact sequence.

Corollary 3.14: The short exact sequence (3) is split when $\mathrm{p}$ does not divide $(\mathrm{n}-4)$.

Proof: Assume $p \nmid(n-4)$. Let $\varphi: S(n-$ $2,2) \rightarrow G$ be define as follows: $\varphi\left(\left(x_{r}-\right.\right.$ $\left.\left.x_{s}\right)\left(x_{t}-x_{l}\right)\right)=\frac{1}{n-4} \sum_{\substack{k=1 \\ k \neq r, s, t, l}}^{n}\left(x_{r} x_{t} x_{k}-\right.$ $\left.x_{r} x_{l} x_{k}-x_{s} x_{t} x_{k}+x_{s} x_{l} x_{k}\right)$.Then for any $\tau \in S_{n}$ we get $\varphi\left(\tau\left(x_{r}-x_{s}\right)\left(x_{t}-x_{l}\right)\right)=$ $\varphi\left(\left(\tau x_{r}-\tau x_{s}\right)\left(\tau x_{t}-\tau x_{l}\right)\right)=$ $\varphi\left(\left(x_{r_{1}}-x_{s_{1}}\right)\left(x_{t_{1}}-x_{l_{1}}\right)\right) \quad=\frac{1}{n-4}$ $\sum_{k_{1}=1}^{n} \quad\left(x_{r_{1}} x_{t_{1}} x_{k_{1}}-x_{r_{1}} x_{l_{1}} x_{k_{1}}-\right.$ $k_{1} \neq r_{1}, s_{1}, t_{1}, l_{1}$ $\left.x_{s_{1}} x_{t_{1}} x_{k_{1}}+x_{s_{1}} x_{l_{1}} x_{k_{1}}\right)=\frac{1}{n-4}$ 
$\sum_{\substack{k_{1}=1 \\ k_{1}^{\neq} \neq r_{1}, S_{1}, t_{1}, l_{1}}}^{n} \tau\left(x_{r} x_{t} x_{k}-x_{r} x_{l} x_{k}-x_{s} x_{t} x_{k}+\right.$

$\left.x_{s} x_{l} x_{k}\right)=\frac{1}{n-4} \tau\left(\sum_{\substack{k=1 \\ k \neq r, s, t, l}}^{n}\left(x_{r} x_{t} x_{k}-\right.\right.$ $\left.\left.x_{r} x_{l} x_{k}-x_{s} x_{t} x_{k}+x_{s} x_{l} x_{k}\right)\right)=\tau \varphi\left(\left(x_{r}-\right.\right.$ $\left.\left.x_{s}\right)\left(x_{t}-x_{l}\right)\right)$. Hence $\varphi$ is a $F S_{n^{-}}$ homomorphism. Moreover we have

$$
\begin{aligned}
& \overline{\bar{d}}_{2} \varphi\left(\left(x_{r}-x_{s}\right)\left(x_{t}-x_{l}\right)\right)= \\
& \overline{\bar{d}}_{2}\left(\frac { 1 } { n - 4 } \sum _ { \substack { k = 1 \\
k \neq r , s , t , l } } ^ { n } \left(x_{r} x_{t} x_{k}-x_{r} x_{l} x_{k}-\right.\right. \\
& \left.\left.x_{s} x_{t} x_{k}+x_{s} x_{l} x_{k}\right)\right) \\
& =\frac{1}{n-4} \sum_{\substack{k=1 \\
k \neq r, s, t, l}}^{n} \overline{\bar{d}}_{2}\left(x_{r} x_{t} x_{k}-x_{r} x_{l} x_{k}-\right. \\
& \left.x_{s} x_{t} x_{k}+x_{s} x_{l} x_{k}\right)=\frac{1}{n-4}\left(( n - 4 ) \left(x_{r}-\right.\right. \\
& \left.\left.x_{s}\right)\left(x_{t}-x_{l}\right)\right)=\left(x_{r}-x_{s}\right)\left(x_{t}-x_{l}\right) \quad \text { Thus } \\
& \overline{\bar{d}}_{2} \varphi=I \text { on } S(n-2,2) \text {. Hence the } \\
& \text { sequence(3) is split when } p \nmid(n- \\
& \text { 4). Moreover } \quad G=\operatorname{ker} \overline{\bar{d}}_{2} \oplus \bar{G} ; \bar{G}= \\
& \varphi(S(n-2,2)) \text {. }
\end{aligned}
$$

Proposition 3.15: $\quad S(n-3,3) \quad$ is a proper $K S_{n}$ - submodule of $G$.

Proof: Since $\quad S(n-3,3)=F S_{n}\left(x_{2}-\right.$ $\left.x_{1}\right)\left(x_{4}-x_{3}\right)\left(x_{6}-x_{5}\right) \quad$ and $G=$ $F S_{n}\left(x_{1} x_{3} x_{6}-x_{1} x_{4} x_{6}+x_{2} x_{4} x_{6}-x_{2} x_{3} x_{6}\right)$ then

$\mathrm{y}=\left(x_{2}-x_{1}\right)\left(x_{4}-x_{3}\right)\left(x_{6}-x_{5}\right)=$ $\left(x_{1} x_{3} x_{6}-x_{1} x_{4} x_{6}+x_{2} x_{4} x_{6}-x_{2} x_{3} x_{6}\right)+$ $\left(x_{1} x_{4} x_{5}-x_{1} x_{3} x_{5}+x_{2} x_{3} x_{5}-x_{2} x_{4} x_{5}\right) \in$ $F$. Thus $S(n-3,3) \subset G$.Moreover since $\overline{\bar{d}}_{2}=\left.\bar{d}_{2}\right|_{G}$, then we get ker $\overline{\bar{d}}_{2} \subset \operatorname{ker} \bar{d}_{2}$ and since $\operatorname{kerd}_{2}=\operatorname{ker} \bar{d}_{2}$. Hence ker $\overline{\bar{d}}_{2} \subset$ $\operatorname{kerd}_{2}$ and by definition of $\overline{\bar{d}}_{2}$ we get $\overline{\bar{d}}_{2}(y)=0$ which implies that $(n-3,3) \subset$ ker $\overline{\bar{d}}_{2} \subset G$. Hence $\mathrm{S}(\mathrm{n}-3,3)$ is a proper $F S_{n}$ - submodule of $G$.

Theorem 3.16: If $p \neq 2,3$ and $p \mid(n-3)$ then we have the following series: 1) $0 \subset F \sigma_{3} \subset F \sigma_{3} \oplus S(n-3,3) \subset F \sigma_{3} \oplus$ ker $\overline{\bar{d}}_{2} \subset F \sigma_{3} \oplus G \subset F \sigma_{3} \oplus M_{0}(n-3,3)$. 2) $0 \subset S(n-3,3) \subset F \sigma_{3} \oplus S(n-3,3) \subset$ $F \sigma_{3} \oplus$ ker $\overline{\bar{d}}_{2} \subset F \sigma_{3} \oplus G \subset F \sigma_{3} \oplus M_{0}(n-$ $3,3)$.
Proof: Since $p \mid(n-3)$, then $\quad p \nmid(n-4)$ and by Corollary (3.19) we get $G=\operatorname{ker} \overline{\bar{d}}_{2} \oplus \bar{G} ; \bar{G}=\varphi(S(n-2,2)) \cong$ $S(n-2,2)$ and by Proposition(3.20) we have $S(n-3,3) \subset \operatorname{ker} \overline{\bar{d}}_{2} \subset G$.

Since $\sigma_{3}(n)=\sum_{1 \leq i<i<k \leq n} x_{i} x_{\mathrm{j}} x_{k}$ and the sum of coefficients of $\sigma_{3}(n)$ is $\frac{n(n-1)(n-2)}{6}$ then $\sigma_{3}(n) \notin M_{0}(n-3,3) \quad$ and $\quad \sigma_{3}(n) \notin G$ which implies that $\sigma_{3}(n) \notin$ ker $\overline{\bar{d}}_{2}$. i.e. $F \sigma_{3} \cap G=0 \quad$ and $\quad F \sigma_{3} \cap k e r \overline{\bar{d}}_{2}=$ $0 \quad$.Hence $F \sigma_{3} \oplus$ ker $\overline{\bar{d}}_{2} \subset F \sigma_{3} \oplus G$ Moreover we have $F \sigma_{3} \oplus S(n-3,3) \subset$ $\mathrm{F \sigma}_{3} \oplus \mathrm{ker} \overline{\bar{d}}_{2}$.Thus we get the following series:

1) $0 \subset F \sigma_{3} \subset F \sigma_{3} \oplus S(n-3,3) \subset F \sigma_{3} \oplus$ ker $\overline{\bar{d}}_{2} \subset F \sigma_{3} \oplus G \subset F \sigma_{3} \oplus M_{0}(n-3,3)$. 2) $0 \subset S(n-3,3) \subset F \sigma_{3} \oplus S(n-3,3) \subset$ $F \sigma_{3} \oplus$ ker $\overline{\bar{d}}_{2} \subset F \sigma_{3} \oplus G \subset F \sigma_{3} \oplus M_{0}(n-$ $3,3)$.

\section{References:}

[Ellers:2007]:Harald Ellers, John Murray, Branching rules for Specht modules, Journal of Algebra 307(2007)278-286.

[Farahat:1962]:H.K. Farahat, On the Natural Representation of the Symmetric groups, Proc .Glasgow Math.Assoc.5 (1962),121-136.

[Joyce:2017]:David Joyce, Clark University, "Introduction to Modern Algebra" Version 1.2.7,5 Dec.2017.

[Kerber:2004]:Addbert Kerber, Axel Kohnert , Modular irreducible representation of the Symmetric group as linear Codes. European Journal of Conbinatorics 25 (2004) 1285-1299.

[Peel:1969]:M.H .Peel, On the Second Natural Representation of the Symmetric groups. Glasgow Math .Journal vol.10 part1(1969),2537.

[Peel:1971]:M.H .Peel, Hook Representation of the Symmetric groups ,Glasgow Math .Journal vol.12 part 2(1971),136-149. 
في التمثيل الطبيعي الثالث

$$
\text { قيّم الرياضي دلفي علي }
$$$$
\text { كلية التربية للعلوم الصرفة }
$$

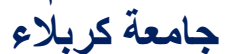

\section{reyadhdelphi@gmail.com}

$$
\begin{aligned}
& \text { علي عبد الصاحب محمد }
\end{aligned}
$$

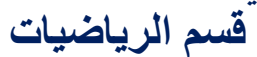

$$
\begin{aligned}
& \text { كلية العلوم } \\
& \text { جامعة التهرين } \\
& \text { alibotahi@gmail.com }
\end{aligned}
$$

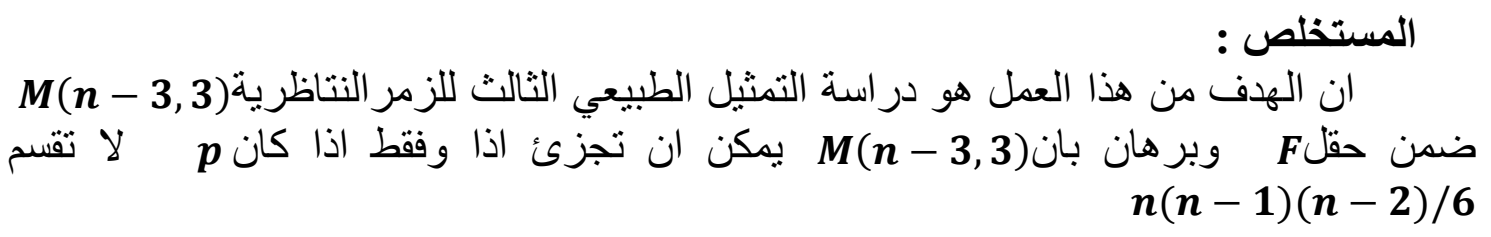

